\title{
Optical Coherence Tomography (OCT): Imaging the Visual Pathway as a Model for Neurodegeneration
}

\author{
Kristin M. Galetta, ${ }^{1}$ Peter A. Calabresi,${ }^{4}$ Elliot M. Frohman,${ }^{5}$ and \\ Laura J. Balcer ${ }^{1,2,3,6}$ \\ ${ }^{I}$ Department of Neurology, ${ }^{2}$ Department of Ophthalmology, ${ }^{3}$ Department of Epidemiology, University of Pennsylvania School \\ of Medicine, Philadelphia, Pennsylvania; ${ }^{4}$ Department of Neurology, Johns Hopkins University School of Medicine, \\ Baltimore, Massachusetts; ${ }^{5}$ Department of Neurology, University of Texas Southwestern Medical Center, Dallas, Texas; \\ ${ }^{6} 3$ E. Gates-Neurology, 3400 Spruce Street, Philadelphia, Pennsylvania 19104
}

\begin{abstract}
Summary: Axonal and neuronal degeneration are important features of multiple sclerosis (MS) and other neurologic disorders that affect the anterior visual pathway. Optical coherence tomography (OCT) is a non-invasive technique that allows imaging of the retinal nerve fiber layer (RNFL), a structure which is principally composed of ganglion cell axons that form the optic nerves, chiasm, and optic tracts. Since retinal axons are nonmyelinated until they penetrate the lamina cribrosa, the RNFL is an ideal structure (no other central nervous system tract has this unique arrangement) for visualizing the processes of neurodegeneration, neuroprotection and, potentially, even neuro-repair. OCT is capable of providing high-resolution reconstructions of retinal anatomy in a rapid and reproducible fashion and permits objective analysis of the RNFL (axons) as well as ganglion cells and other neurons in the macula. In a systematic OCT examination of multiple sclerosis (MS) patients, RNFL thickness and macular volumes are reduced when compared to disease-free controls.
\end{abstract}

Conspicuously, these changes, which signify disorganization of retinal structural architecture, occur over time even in the absence of a history of acute demyelinating optic neuritis. RNFL axonal loss in MS is most severe in those eyes with a corresponding reduction in low-contrast letter acuity (a sensitive vision test involving the perception of gray letters on a white background) and in those patients who exhibit the greatest magnitude of brain atrophy, as measured by validated magnetic resonance imaging techniques. These unique structure-function correlations make the anterior visual pathway an ideal model for investigating the effects of standard and novel therapies that target axonal and neuronal degeneration. We provide an overview of the physics of OCT, its unique properties as a non-invasive imaging technique, and its potential applications toward understanding mechanisms of brain tissue injury in MS, other optic neuropathies, and neurologic disorders. Key Words: Optical coherence tomography, retina, optic nerve, multiple sclerosis, vision.

\section{INTRODUCTION}

Axonal and neuronal degeneration are important features of multiple sclerosis (MS) and other neurologic disorders that affect the anterior visual pathway [1-5]. Optical coherence tomography (OCT) is a noninvasive technique that allows imaging of the retinal nerve fiber layer (RNFL), a structure that contains ganglion cell axons which form the optic nerves, chiasm, and optic tracts (FIGs. 1, 2) [5-32].

Electronic supplementary material The online version of this article (doi:10.1007/s13311-010-0005-1) contains supplementary material, which is available to authorized users.

Address correspondence and reprint requests to: Laura J. Balcer, M.D., M.S.C.E., 3 E. Gates-Neurology, 3400 Spruce Street, Philadelphia, PA 19104. E-mail: lbalcer@mail.med.upenn.edu; laura.balcer@uphs. upenn.edu.
Since these axons are nonmyelinated within the retina, the RNFL is an ideal structure with which to visualize the processes of neurodegeneration, neuroprotection and, potentially, even neuro-repair. In contrast to the peripapillary RNFL, which contains axons, the macula contains a large proportion of retinal ganglion cell (RGC) neurons (about 34\% of total macular volume) (FIG. 3) [21]. About $1200 \mu \mathrm{m}$ in diameter, the macula is easily identified within the retina. The application of non-conventional magnetic resonance imaging (MRI) techniques, such as magnetization transfer imaging, magnetic resonance spectroscopy, and diffusion tensor imaging, has led to only modest achievements in linking imaging data with clinical measures of disease severity for neurologic disorders in general and for multiple sclerosis (MS) in particular. Alternately, OCT has been incorporated 


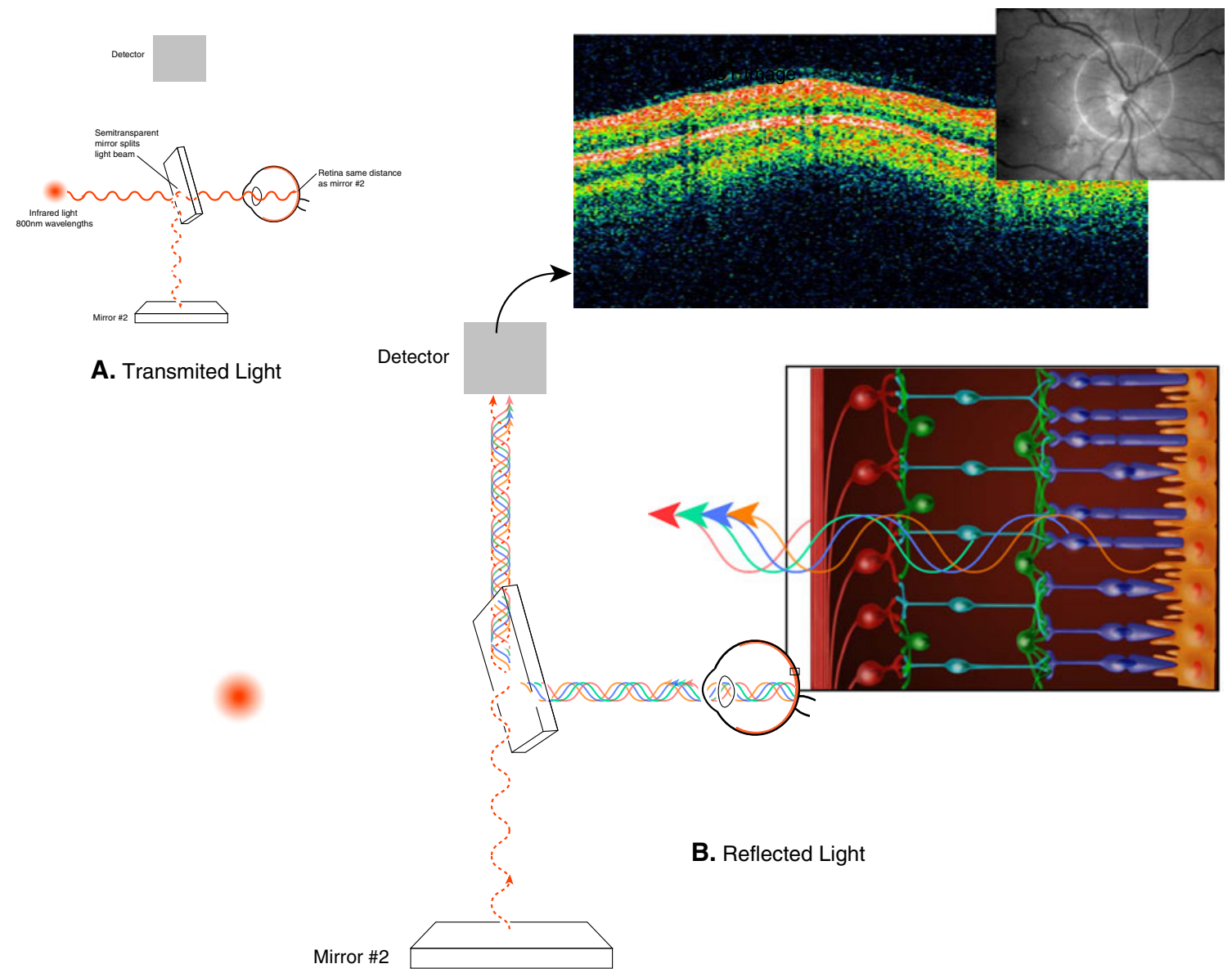

FIG. 1. Images of the internal retinal structure taken with optical coherence tomography (OCT), demonstrating the processes involved in using this technology. (a) Low-coherence infrared light is transmitted into the eye through use of an interoferometer. (b) The infrared light is transmitted through the pupil and then penetrates through the transparent nine layers of the retina. The light subsequently backscatters and returns through the pupil, where detectors can analyze the interference of light returning from the layers of the retina compared with light traveling a reference path (mirror \#2). An algorithm mathematically uses this information to construct a gray-scale or false-color image representing the anatomy of the retina (shown in the upper right portion of the figure). (c) A fundus image from the OCT device, showing the optic disc appropriately centered and surrounded by the target image circumference marker for analysis of the retinal nerve fiber layer. Reproduced with permission from Frohm et al. [5]. (High resolution version of this image is available in the electronic supplementary material.)

with increasing frequency as an exploratory outcome in treatment trials, observational studies, and even clinical practice in order to achieve a greater understanding of the relationship between changes in retinal structure and patient reported outcomes of visual function [33-36].

This review describes a number of advancements in the development of OCT as a novel technology that enables objective analysis of the processes of neurodegeneration within a highly discrete and eloquent central nervous system structure-the retina. OCT enables investigators to rapidly and reproducibly evaluate the structural composition of the retina. Ultimately, OCT could substantially increase our understanding of the mechanisms of tissue injury in MS, be used to identify new therapeutic strategies focused on neuroprotection of central axonal and neuronal structures, and also enable the detection and monitoring of the processes of neurorestoration, a treatment goal not yet within the capability of the neurologist [23-28].

\section{FEATURES AND ADVANTAGES OF OCT IMAGING}

OCT was first reported by Huang et al. [37] in 1991. In vivo retinal imaging was first demonstrated in 1993 [38, 39], and early studies in 1995 provided the first demonstration of OCT imaging of the normal retina [40] and of macular pathology [41]. OCT is the optical analog of ultrasound B-mode imaging (Table 1) [5, 24-28]. With OCT, high-resolution cross-sectional or 3-dimensional images of the internal retinal structure are generated by an optical beam being scanned across the retina and the magnitude and echo time delay of backscattered light being measured (FIG. 1) [5, 24-28]. In contrast to ultrasound, direct 


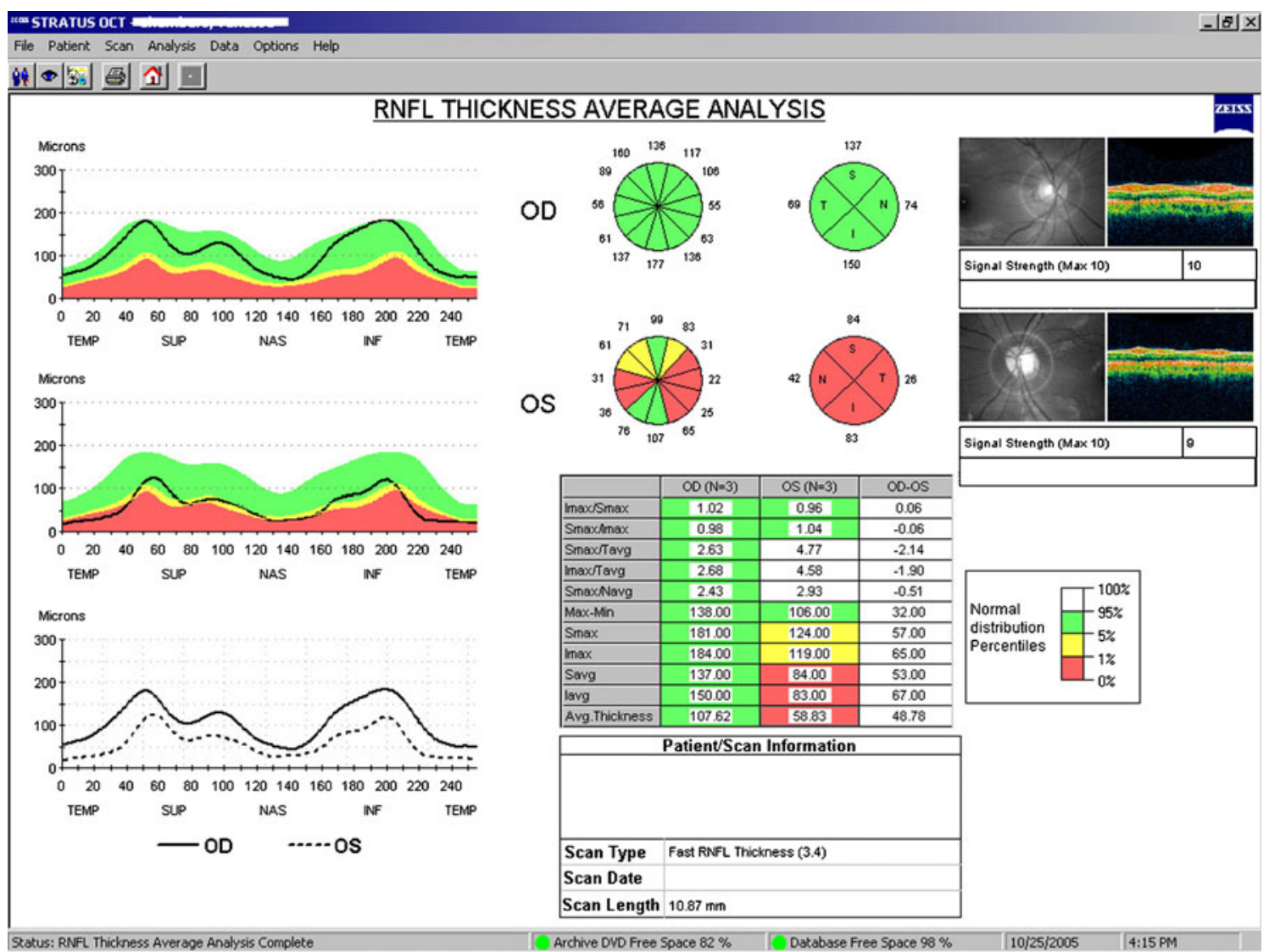

FIG. 2. A typical OCT report from a patient with multiple sclerosis (MS), generated by Zeiss Stratus OCT3TM with software ver. 4.0 (Carl Zeiss Meditec, Jena, Germany). On the upper left, the retinal nerve fiber layer (RNFL) thickness is plotted (y-axis) with respect to a circumferential retinal map on the $x$-axis [temporal-superior-nasal-inferior-temporal (TSNIT) quadrants of the RNFL]. Note the normal 'double-hump' appearance of the topographic map of the right eye (OD), signifying the thicker RNFL measures derived from the superior and inferior retina compared with the nasal and temporal regions. Also note the quadrant and clockface sector measures of RNFL thickness (upper middle illustration). The table (lower middle) compiles the quantitative data, including the average RNFL thickness (bottom row). This patient experienced an episode of left optic neuritis 6 months before this study. Note the marked reduction in RNFL thickness across all quadrants (red region denoting values $<1 \%$ of what would be expected in a reference population), multiple sectors, and with respect to the average RNFL thickness (bottom row of table). TEMP $=$ Temporal; SUP = superior; NAS = nasal; INF = inferior; OD = right eye; OS = left eye. Reproduced with permission from Frohman et al. [5]. (High resolution version of this image is available in the electronic supplementary material.)

detection of light echoes is not possible in OCT because the speed of light is much faster than the speed of sound. Early OCT systems were based on low coherence interferometry, a phenomenon demonstrated by Sir Isaac Newton. In this technique, measurements are performed using a Michelson type interferometer with a low-coherence-length, superluminescent diode light source. One arm of the interferometer directs light onto the sample and collects the backscattered signal. A second reference arm has a reflecting mirror, which is mechanically scanned to vary the time delay and measure interference. The use of a low-coherence-length light source means that interference occurs only when the distance traveled by the light in the sample and reference arms of the interferometer match to within the coherence length. This enables the echo delays of the light from the tissue to be measured with extremely high temporal accuracy. The resulting data set is a 2dimensional or 3-dimensional array, which represents the optical backscattering in a cross section or volume of the tissue. These data can be processed and displayed as a 2-dimensional or volumetric gray-scale or false-color image (Table 1) [5].

Recently, the performance of OCT has been improved by substantial technological advances [42]. The development of 'Fourier domain' (or spectral domain) detection, a technique first proposed in 1995, has in particular enhanced ophthalmic OCT technology. Fourier domain OCT detects light echoes by measuring the interference spectrum from an interferometer with a stationary reference arm. A spectrometer and high-speed line-scan camera record the interference spectrum, which is Fourier transformed to obtain 


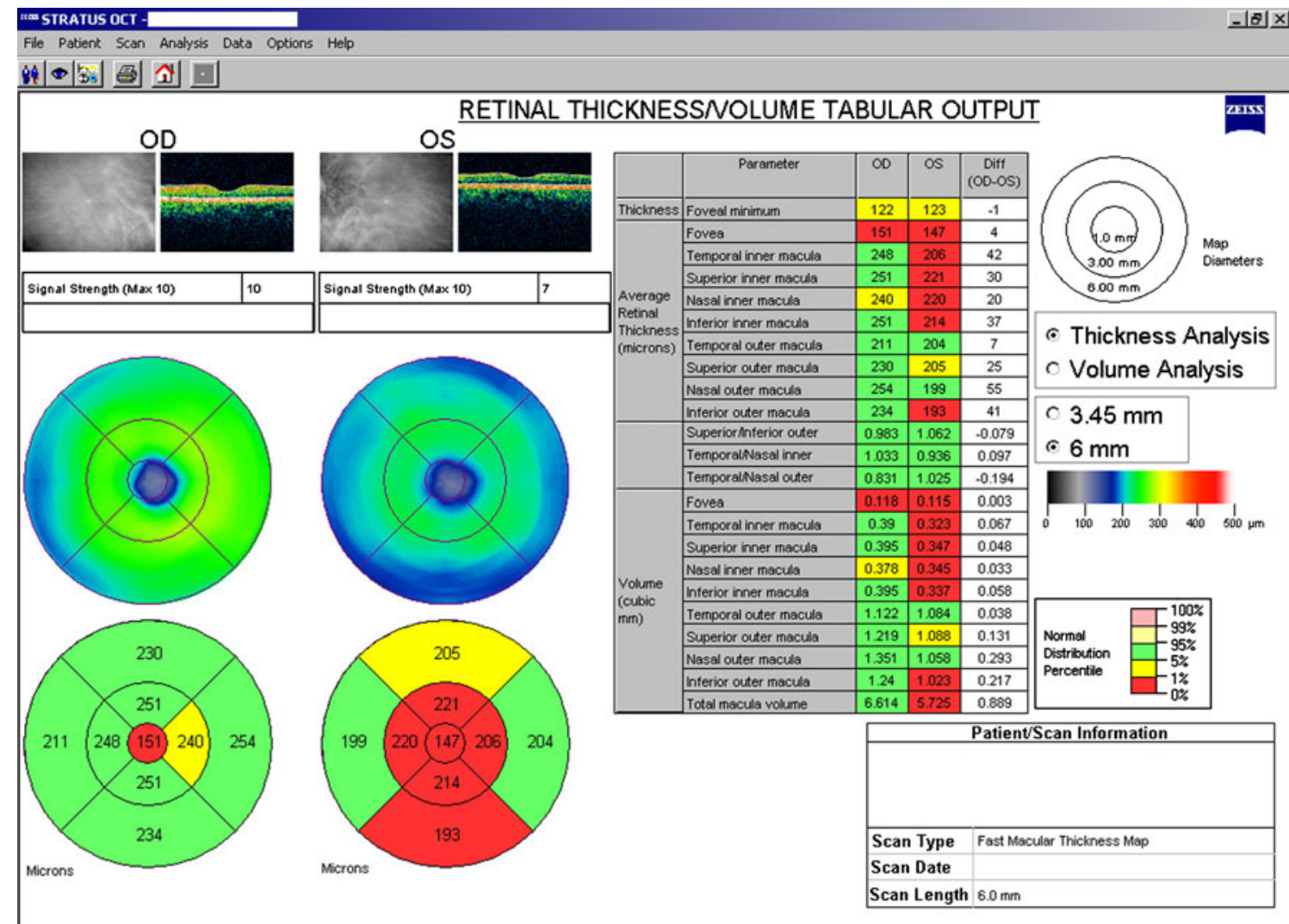

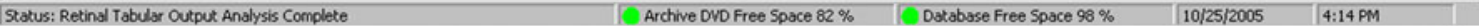

FIG. 3. An OCT report for the macular region of the retina from the same patient with MS as is shown in FIG. 2. Note the volume reductions in the foveola (central macula) and the parafoveal quadrants on the left of the report. Whereas the reductions in RNFL thickness implicate the loss of ganglion cell axons, macular changes implicate losses of the ganglion cell neurons themselves. While the patient has had no history of optic neuritis in the right eye, there are some subtle macular changes on that side, suggesting occult involvement of this eye as well. Reproduced with permission from Frohman et al. [5]. (High resolution version of this image is available in the electronic supplementary material.)

the magnitude and echo time delay of the light (the axial scan). Fourier domain OCT detects all light echoes simultaneously, leading to a dramatic increase in sensitivity that enables high-speed imaging [43, 44]. Retinal imaging with Fourier domain OCT became possible only with recent advances in camera technology. The first retinal images with this technique were reported in 2002 [45], and high-speed, high-resolution retinal imaging was demonstrated in 2003 [46, 47]. The technology became commercially available in 2006; most commercial instruments achieve an axial image resolution of 5-7 $\mu \mathrm{m}$ with imaging speeds of 25,000 axial scans/s, which is approximately 50 -fold faster than the previous generation of time domain OCT technologies (FIGS. 4, 5) [5, 24-28, 39-48].

Retinal imaging technologies such as OCT can rapidly evaluate the integrity of the RNFL and the macula for the purpose of tracking disease progression and, potentially, also neuroprotection. Studies have demonstrated that thinning of the RNFL (assessed by average, quadrant, and clockface sector analyses) and volume reductions in the macula are associated with stereotypic declines in visual functioning, as measured by low-contrast letter acuity and visual field analyses (Table 1) [5-9, 11, 14, 24-28, 31].

\section{MULTIPLE SCLEROSIS AND OPTIC NEURITIS AS SPECIFIC MODELS FOR VISUAL PATHWAY AXONAL LOSS}

A direct bedside visualization of the retina and optic nerve was first achieved in 1851 following the introduction of the hand-held ophthalmoscope by Helmholtz [49]. Over a century later, in 1974, Frisén and Hoyt [50] reported their subjective analysis of thinning of the RNFL (which is principally composed of axons from ganglion cell neurons) in patients with MS, as evaluated with hand-held ophthalmoscopy. This observation was corroborated in a postmortem study that demonstrated atrophy of the RNFL (typically containing about $80 \%$ axons and $20 \%$ glia) in 35 of 49 eyes of patients with MS [51]. Nevertheless, this 
Table 1. Features and Advantages of Optical Coherence Tomography Imaging

Physical properties

1. Optical analog of ultrasound

2. Generates cross-sectional images measuring backreflected echoes of light

3. Interferometric methods used to detect echoes since times are too fast for direct detection

4. Current technology has $8-10 \mu \mathrm{m}$ resolution; new technology has 5-7 $\mu \mathrm{m}$ resolution

Imaging metrics (current technology)

1. Average retinal nerve fiber layer (RNFL thickness)

2. Quadrant and clockface sector analyses

3. Total macular volumes

4. Quadrant assessment of parafoveal areas

5. Inferior and superior RNFL are thickest [double hump waveform on temporal-superior-nasal-inferior-temporal (TSNIT) analyses]

Imaging metrics (new technology)

1. RNFL mapping

2. Mapping intraretinal layers in the macula

3. 3D-OCT of optic nerve head topography and internal structure

4. Technology is new and metrics remain to be defined

Biomarker features and validation

1. Correlates with high-contrast and low-contrast visual acuity

2. Pathological distribution predicts visual field changes

3. OCT metrics predict brain atrophy

4. Subtypes of multiple sclerosis predict severity of RNFL thinning

5. Measures of laser polarimetry with variable corneal compensation (GDx-VCC) corroborate OCT evidence of axonal degeneration

Application features

1. Testing performance is quick and easy

2. Pupil dilation is typically not required

3. Low coefficient of variation for repeated measures

4. Low interindividual and intra-individual variation

5. Low variability across different centers using the same device

6. Scan quality can be assured with disc centering and adequate signal strength

Adapted from Frohman et al. [5] with permission.

RNFL $=$ Retinal nerve fiber layer; OCT $=$ optical coherence tomography; $3 \mathrm{D}=3$-dimensional.

study was not sufficiently quantitative to enable a full appreciation of the relationship between vision, RNFL thickness, and the integrity of the approximately 1.2 million axons that make up the optic nerve.

Recent years has seen the emergence of 'quantitative ophthalmoscopes' such as Heidelberg Retinal Tomography (HRT), laser polarimetry with variable corneal compensation (GDx-VCC), and OCT, all of which can be used to efficiently and objectively measure changes in structural architecture within the retina [5, 29, 52]. Measures derived from these modalities have been shown to directly and reliably relate to visual function and seem to parallel the pathological changes that occur in the brains of patients with MS.
Recent studies have thus demonstrated that MS and optic neuritis $(\mathrm{ON})$ provide useful clinical models with which to couple clinical measures of visual function with validated and objective structural and physiological correlates of MS $[5,24-28,36]$. The anterior visual system is such a frequent target of the MS disease process that, on postmortem analysis, almost all patients with MS will be found to have characteristic changes in the retina and optic nerve, regardless of whether they have previously experienced acute ON $[2,24-28,53$, 54]. In addition, as many as $30-70 \%$ of patients with MS will, in fact, have ON during the course of their disease $[55,56]$. These data suggest that the visual system has a very high predilection for developing disease-related disability and could be used effectively to illustrate the histopathology of the disease process in MS.

In contrast with occult optic neuropathy in MS, which is a largely subclinical manifestation of disease in the anterior visual system, most cases of acute $\mathrm{ON}$ are associated with pain and visual disturbances, including diminished vision, color desaturation, poor low-contrast acuity and sensitivity, and field abnormalities [55]. ON can, therefore, be evaluated with clinical and neurophysiological techniques, such as patient-reported acuity (high and low contrast) and sensitivity, visual field analysis, and visual evoked responses $[55,56]$. Furthermore, in one study, coronal, fat-suppressed, T1-weighted gadolinium-enhanced MRI revealed enhancement in the optic nerve in over $90 \%$ of cases of optic neuritis (ON), confirming a breach in the integrity of endothelial tight junctions at the blood-brain barrier [57]. Studies with conventional MRI have shown that the optic nerve area declines subsequent to an event of $\mathrm{ON}$ when compared with the unaffected (or at least not acutely affected) eye [58]. Retinal imaging techniques have added greatly to our knowledge of this area, revealing changes in retinal architecture that reflect alterations in visual system physiology and function [5, 24-28].

Visual function has been shown to be directly related to the integrity of retinal anatomy. In particular, abnormalities in this complex structure following ON produce corresponding clinical deficits in patient performance on vision tests [29]. However, since visual function is a measure of both anterior and posterior visual pathways, novel imaging approaches that are specific to the retina and optic nerve may enable more specific quantification of axon injury related to ON [59].

\section{ROLE FOR OCT IN MODELING AXONAL AND NEURONAL LOSS IN MS}

The earliest application of OCT technology to the study of MS was reported by Parisi et al. [6] in 1999. In this study, which utilized first-generation OCT 


\section{a}

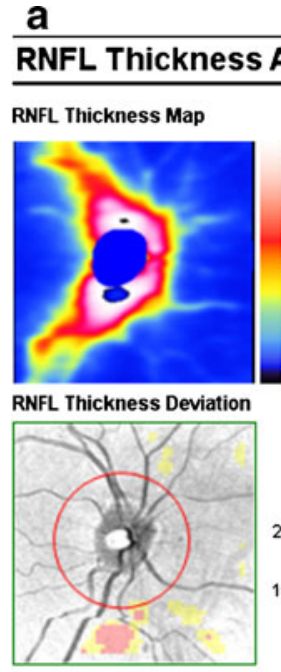

Offset $(-0.33 ; 0.09) \mathrm{mm}$ RNFL TSNIT Normative Data
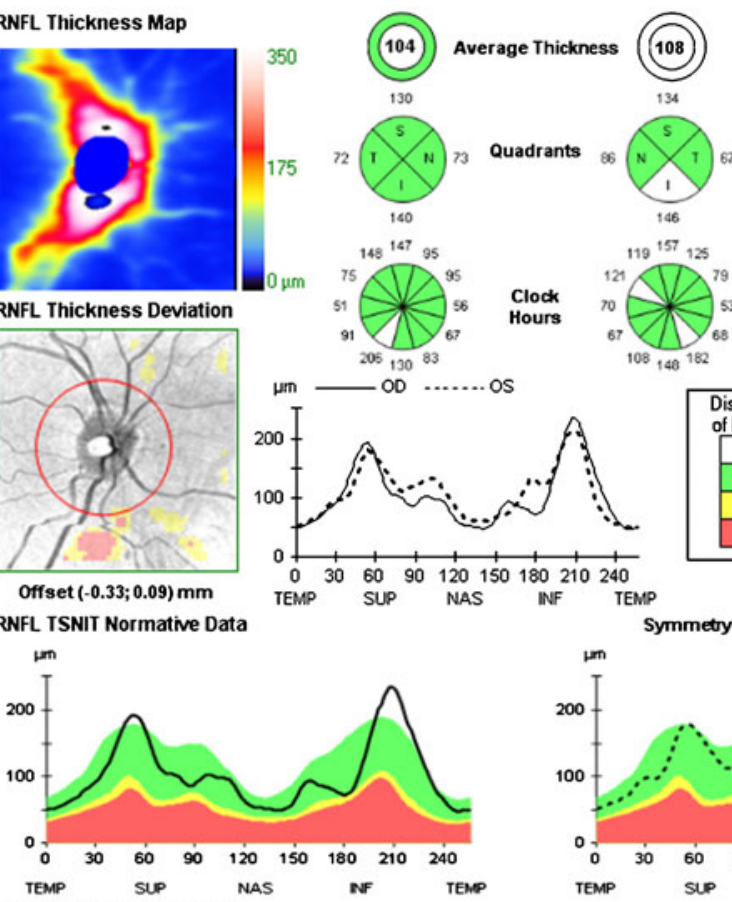

un $-O D \ldots . . .0$ OS

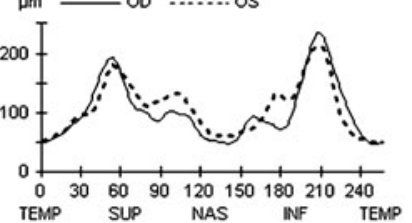

$119^{157} 12$

Clock Hours
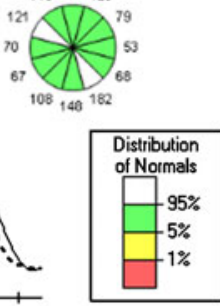

Symmetry
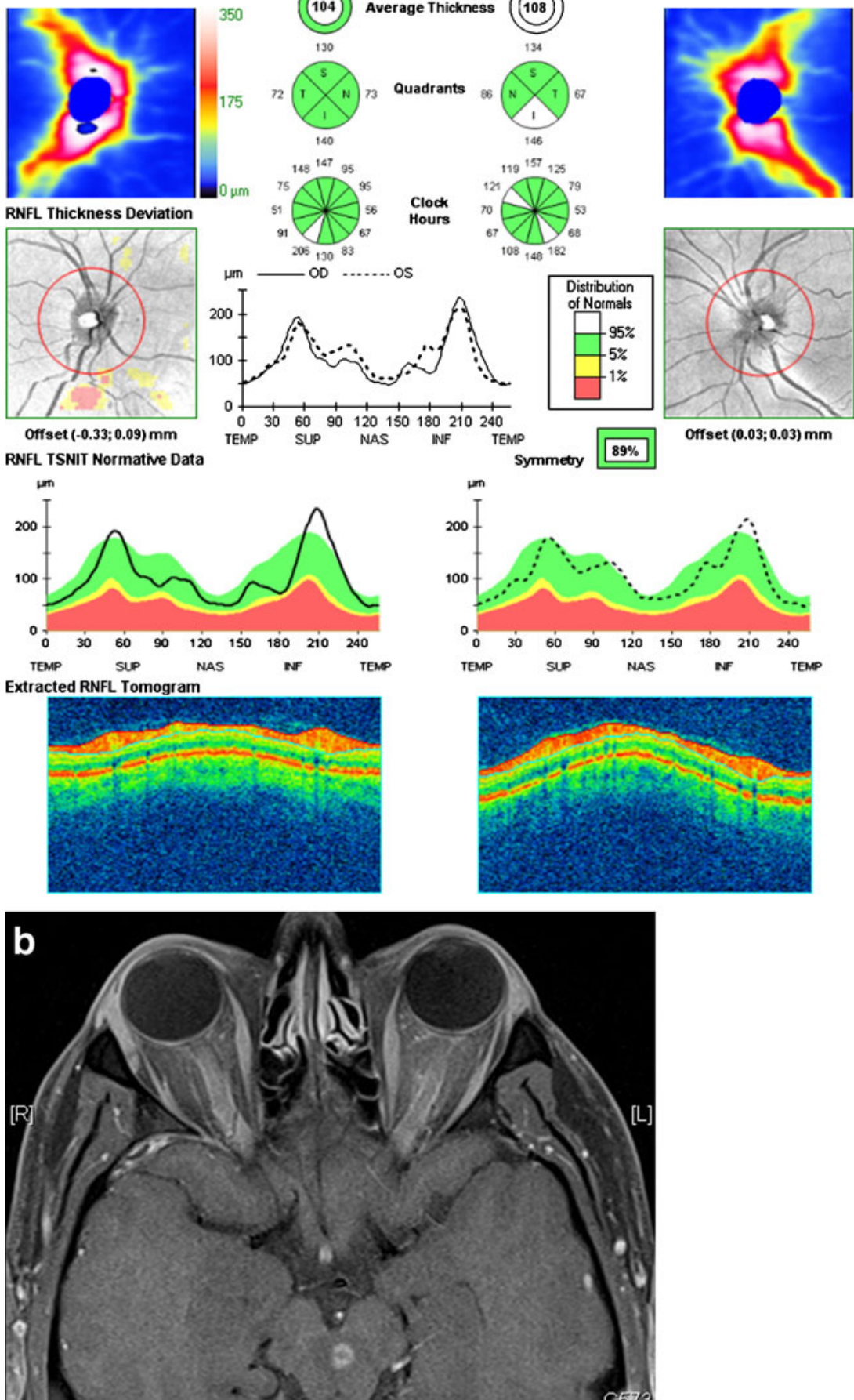

FIG. 4. (a) Example of a high-resolution Fourier domain OCT report from a patient with neuromyelitis, generated by Zeiss Stratus Cirrus high-resolution Fourier domain OCT (Carl Zeiss Meditec). The patient had bilateral mild disc hyperemia and edema in the setting of subacute visual loss. (b) Magnetic resonance image of the orbits with fat-saturation and gadolinium showed bilateral optic nerve enhancement and an enhancing lesion in the midbrain. (High resolution version of this image is available in the electronic supplementary material.)

technology, 14 patients with MS who had completely recovered from a previous event of acute ON were analyzed. The thickness of the RNFL was shown to be reduced by $46 \%$ in the affected eyes of the patients with MS versus the control eyes $(p<0.01)$, and by $28 \%$ when affected eyes were compared with the 'unaffected' eyes of the same patient $(p<0.01)$. Even in the supposedly unaffected eyes of the patients, 


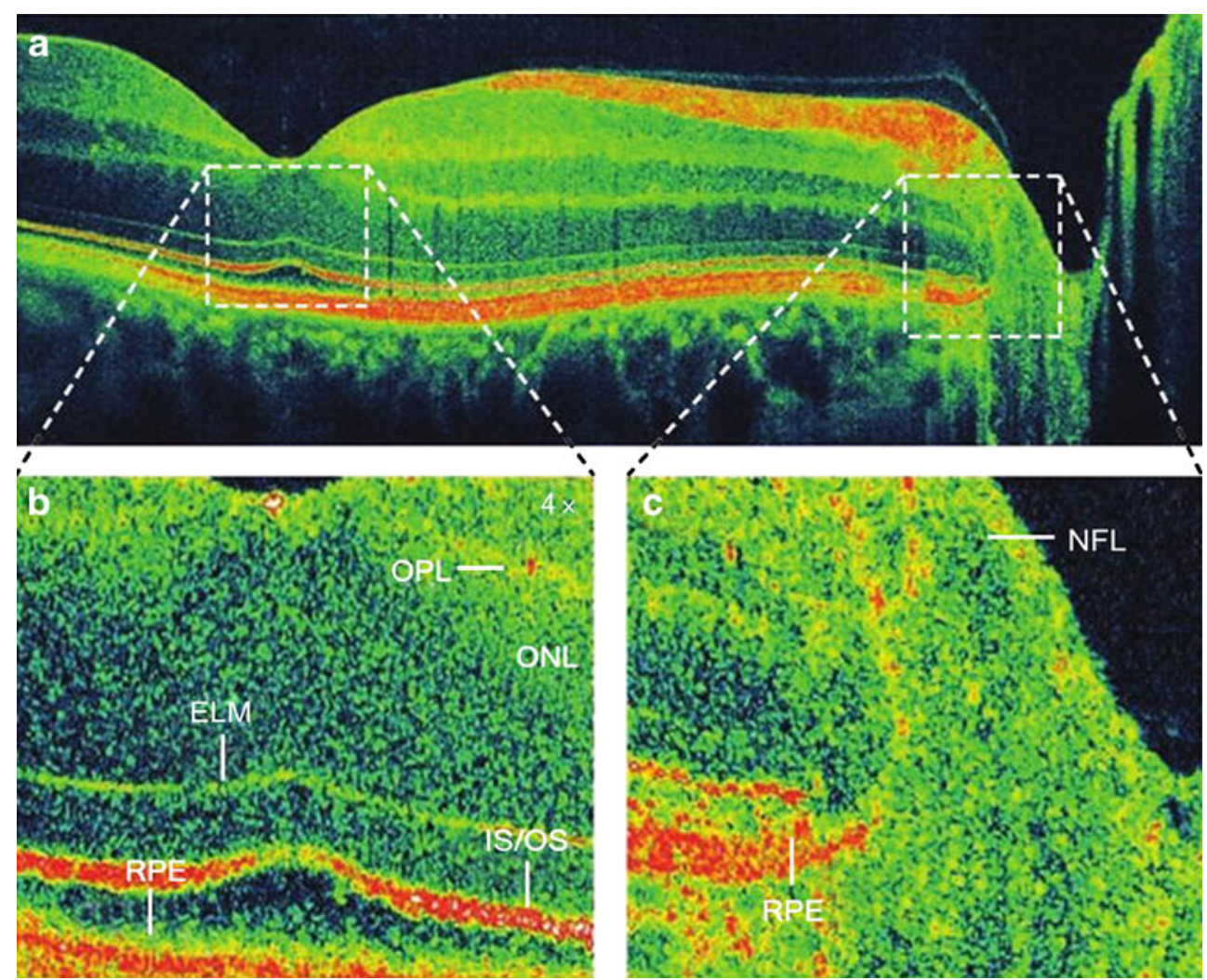

FIG. 5. High-definition, ultra-high-resolution OCT scans. The high data acquisition speeds available with spectral domain detection enable the acquisition of high-definition images with large numbers of transverse pixels. (a) A 10,000 axial scans per second image of the papillomacular axis acquired in $0.6 \mathrm{~s}$. The axial image resolution is approximately $3 \mu \mathrm{m}$. The image may be zoomed in the foveal (b) or optic disc regions (c) to visualize details of internal retinal morphology. OCT has been termed "optical biopsy", and ultra-high-resolution OCT imaging can provide excellent visualization of retinal architecture. ELM = External limiting membrane; IS/OS = junction between inner and outer photoreceptor segments; NFL = nerve fiber layer; ONL = outer nuclear layer; OPL $=$ outer plexiform layer; RPE $=$ retinal pigment epithelium. Adapted from Drexler and Fujimoto [42] () 2008, Elsevier. (High resolution version of this image is available in the electronic supplementary material.)

however, there was a $26 \%$ reduction in RNFL thickness in comparison to the control eyes $(p<0.01)$.

In 2005, Trip and colleagues [7] reported their observations with OCT in 11 patients with MS and 14 patients with clinically isolated syndrome, with all individuals having a history of a single episode of ON. The study was a cross-sectional analysis with followup ranging from 1 to 9 years after the $\mathrm{ON}$ event. Corroborating the previous findings by Parisi et al. [6] the investigators found a 33\% reduction in RNFL thickness in the eyes of the patients when compared with the eyes of matched controls, and a $27 \%$ reduction when the affected and unaffected eyes of the same patient were compared $(p<0.001)$. Trip et al. [7] extended the utility of OCT by also showing that the macular volume (a reflection of RGC neuronal integrity) was reduced by $11 \%$ in the eyes of patients with a history of $\mathrm{ON}$ when compared with control eyes $(p<0.001)$, and by $9 \%$ in the affected versus the unaffected eye of the same patient $(p<0.001)$.

An important finding of these studies was that the apparently unaffected eyes of patients with MS were in fact significantly abnormal when compared with the eyes of matched control individuals, but were less abnormal than the eye of a patient with a history of ON. This finding indicates that $\mathrm{ON}$ signifies accelerated and more severe histopathological consequences on the retina, in contrast to a more insidious and less perceptible change in individuals without a history of an acute syndrome, in whom the disease process probably more closely resembles glaucoma.

As a validation of the axonal basis of OCT metrics in the RNFL, Trip and colleagues [7] showed that such measures correlated better with visual evoked potential P100 amplitudes (a measure of axonal integrity or function) than with P100 latency (typically a reflection of myelin integrity). It is now generally accepted that disruption of myelin has a direct impact on the function and preservation of axons [60]. During the pathological process, however, these two structural elements can become temporarily uncoupled. The axon cylinder might be intact - albeit vulnerable - for a short period during inflammation and myelin disruption. A demyelinated but intact axon has a number of potential destinies: 1) the 
axon can be remyelinated if viable adult oligodendrocytes in the vicinity can provide new concentric lamellar internodes, or 2) recovery can also be provided by oligodendrocyte progenitors terminally differentiating into adult process-bearing myelinating cells. Even partial remyelination might be protective.

In 2006, Costello and colleagues [8] reported that the majority of patients with MS who have ON (about $75 \%$ ) will sustain 10-40 $\mu \mathrm{m}$ of RNFL loss within a period of only about $3-6$ months following the initial inflammatory event. This finding is striking given that the RNFL is only about $110-120 \mu \mathrm{m}$ thick by the age of 15 years and that most individuals (without a history of glaucoma or macular degeneration) will lose only about $0.017 \%$ per year in retinal thickness, which equates to approximately 10-20 $\mu \mathrm{m}$ over a 60-year period [61]. Costello et al. [8] also provided compelling evidence identifying an injury threshold within the RNFL of about $75 \mu \mathrm{m}$; thinning of the RNFL below this level led to a corresponding decay in visual function, as measured by automated perimetry.

The development of validated measures of visual functioning has greatly facilitated the exploration for a structural marker for neurodegeneration in MS. Balcer et al. [62] and Baier et al. [63] have utilized performance on low-contrast letter acuity charts to compare retinal structure with visual function in patients with MS who have MS pathology in the anterior visual system. Patients with MS and a history of ON will have lower thickness measures of the RNFL than patients with MS but no prior history of $\mathrm{ON}$, or than individuals without a history of either condition. The fact that the severity of visual loss (as confirmed by performance on automated perimetry or low-contrast letter acuity) is a predictor of abnormal retinal architecture is further evidence that OCT can be used as a noninvasive approach to monitor the course of disease in patients with MS and to detect and monitor the efficacy of new therapies targeting mechanisms that might promote neuroprotective effects on retinal axons and ganglion cell neurons (FIG. 2, Table 1) [5].

Axonal and neuronal degeneration might be readily expected to be present in eyes with a known history of $\mathrm{ON}$, but in recent studies, researchers utilizing OCT to examine RNFL thickness in heterogeneous MS cohorts have also considered how scores for low-contrast letter acuity might also reflect RNFL thinning in the eyes of patients with MS who do not have a history of ON [9]. Among 90 patients (180 eyes) and 36 MS-free control individuals (72 eyes), the average RNFL thickness was significantly reduced in the eyes of patients with MS. As expected, eyes from patients with a known history of one or more attacks of ON had significantly lower RNFL thickness $[85 \pm 17 \mu \mathrm{m}( \pm$ standard deviation $)]$ than did the eyes of patients with MS who did not have a prior ON history $(96 \pm 14 \mu \mathrm{m} ; p<0.001)$. RNFL thickness was also reduced in the eyes of patients with MS without an ON history compared with normal control eyes $(105 \mu \mathrm{m}$; $p=0.03)$. With use of normative data included in the OCT 4.0 software (Stratus; Zeiss Humphrey Systems, Dublin, CA) for the OCT 3 Stratus, only 40 (22\%) of 180 eyes from patients with MS had 'abnormal' average RNFL thickness. In view of the fact that the OCT 4.0 normative database considers the fifth percentile for age to be the cutoff for abnormal values, however, abnormalities in RNFL thickness are likely to be of substantially greater prevalence in the eyes of patients with MS and ON.

Results from this study also suggest that the 'unaffected' eyes of patients with a history of ON are at a similar risk for axonal loss as the eyes of patients with MS in general; both MS groups have more axonal loss than age-matched control individuals [9]. Low-contrast letter acuity scores were also found to be significantly correlated with overall average RNFL thickness in the eyes of patients with MS $(p<0.001$ by use of generalized estimating equation models accounting for age and adjusting for within-patient, inter-eye correlations); for every one-line change in low-contrast letter acuity score, an average RNFL thickness difference of $4 \mu \mathrm{m}$ was noted. Average overall RNFL thickness also declined with increasing degrees of overall neurological impairment and disability in the MS cohort, and it was significantly associated with the Expanded Disability Status Scale score and disease duration $(p=0.03)$.

It has now been shown that an RNFL thickness stratification can be achieved when MS is segregated into its various subtypes (Table 1) [5, 11, 13]. In particular, patients with greater brain atrophy, as measured by validated metrics (such as the brain parenchymal fraction), have a more substantial retinal involvement, as measured by RNFL loss [14, 64]. Thus, the eye would seem to be able to accurately model the mechanisms of neurodegeneration and could even be used to detect and monitor neuroprotection in MS. Specifically, OCT can be utilized to measure the integrity of both neurons and their axonal projections within the retina. Further, the severity of retinal damage directly correlates with visual dysfunction clinically, and with both the severity of MS-related brain pathology (i.e., atrophy) and MS clinical subtype designations (as stratified by disease progression status; Table 1) [5].

While prior studies have emphasized the association between worse visual function and reductions in OCTmeasured RNFL thickness [9] and macular volume [21] at a single time point, longitudinal studies have also demonstrated that thinning of the RNFL occurs over time in MS, even among eyes with no history of acute ON [31]. Patients at three academic centers underwent OCT3 (Stratus) measurement of RNFL thickness at 6-month intervals during a mean follow-up of 18 months. Vision 
was assessed using low-contrast letter acuity (2.5\%, $1.25 \%$ contrast) and high-contrast visual acuity. Among 593 eyes of 299 MS patients ( $\geq 6$ months follow-up), eyes with visual loss showed greater RNFL thinning than eyes with stable vision (low-contrast acuity, 2.5\%, $p<0.001$; visual acuity, $p=0.005)$. RNFL thinning increased over time, with average losses of $2.9 \mu \mathrm{m}$ at $2-3$ years and $6.1 \mu \mathrm{m}$ at $3-4.5$ years $(p<0.001$ vs $0.5-1$-year follow-up interval). These patterns were observed for eyes with or without a prior history of ON. Proportions of eyes with RNFL loss greater than test-retest variability $(\geq 6.6 \mu \mathrm{m})$ increased from $11 \%$ at $0-1$ year to $44 \%$ at $3-4.5$ years $(p<0.001)$. This study thereby provides compelling evidence that progressive RNFL thinning occurs as a function of time in MS, even in the absence of a history of $\mathrm{ON}$, and is associated with clinically significant visual loss. These findings are consistent with sub-clinical axonal loss in the anterior visual pathway in MS and support the use of OCT and low-contrast acuity as methods to evaluate the effectiveness of putative neuroprotection protocols [31].

Reproducibility is an important aspect of longitudinal investigations involving OCT technologies, including clinical trials and observational studies. Previous work by Cettomai et al. [16] showed good levels of inter-rater and test-retest reliability for OCT 3 (Stratus) measurements of RNFL thickness in patients with MS and disease-free controls. A more recent study, however, demonstrated that high-resolution Fourier domain OCT techniques afford an even greater degree of reproducibility of RNFL thickness and macular volume assessments [32]. This prospective study of inter-visit, interrater, and intra-rater reproducibility was performed using Cirrus high-resolution OCT. Among 58 patients with MS and 32 healthy controls, there was excellent reproducibility of average and quadrantic RNFL thickness values, average macular thickness, and total macular volume; intraclass correlation coefficients ranged from 0.92 to 0.97 for inter-visit, 0.94 to 0.99 for inter-rater, and 0.83 to 0.99 for intra-rater reproducibility. The authors suggested that, in addition to implementing highresolution Fourier domain technology, the utilization of specific procedures, such as the reading of algorithms and quality control, can serve to optimize the quality of OCT data [32].

While MS is classically considered to be a disorder involving the white matter of the central nervous system, recent pathologic and MRI studies of brain tissue have confirmed that neuronal apoptosis and gray matter degeneration do occur in MS. The retina has proven to be no exception to these findings, and the results of a recent postmortem analysis of 82 patients demonstrated retinal atrophy with shrunken neurons [65]. In patients with MS, there was a dropout of RGC in $79 \%$ of eyes and inner nuclear layer atrophy (amacrine and bipolar cells) in $40 \%$ of eyes. The severity of the retinal atrophy was significantly associated with postmortem brain weight, with a trend towards an association with disease duration. This report by Green and colleagues [65] provides the first description of inner nuclear layer cell loss in MS and supports a role for forthcoming OCT studies that incorporate segmentation technologies to determine the thicknesses of specific retinal layers and structures.

Electrophysiologic measures of the visual pathway, including visual evoked potentials (VEPs), are generally regarded as complementary to clinical measures of function and structural measures of axonal and neuronal integrity. Naismith et al. [66] systematically evaluated the utility of OCT and VEPs to detect the presence of clinical and subclinical $\mathrm{ON}$ and examined the relation of these measures to visual function. This retrospective, crosssectional study evaluated 65 subjects ( $n=96$ eyes) with MS $(n=40)$, clinically isolated syndrome $(n=1)$, neuromyelitis optica (NMO, $n=20$ ), and idiopathic demyelination $(n=4)$. Patients had at least one episode of ON $\geq 6$ months prior to enrollment. VEPs detected ON in $81 \%$ of patients $(32 \%$ of subclinical ON in unaffected eyes and $75 \%$ of all subclinical ON). In contrast, using their criteria, they found that OCT identified $60 \%$ of eyes with ON and less than $20 \%$ of subclinically affected eyes. The authors concluded that OCT is 'less sensitive than VEPs in optic neuritis.' On the other hand, to the extent that this study focused on patients and eyes with relatively poor vision, there were likely floor effects with regard to OCT-determined RNFL thickness.

\section{THE FUTURE OF RETINAL IMAGING IN MS}

The hypothesis that measures of the RNFL are causally related to visual performance has been corroborated in recent experiments by our group utilizing another type of quantitative ophthalmoscope, GDx-VCC (Table 1) [5]. GDx, which is based on the projection of polarized light and its retardation when propagating through a birefringent medium (such as the RNFL), can be used to measure both the thickness and the integrity of the nerve fiber layer [17]. The physical properties of this technology enable the retinal substructure to be examined in order to assess the integrity of axonal microtubules. While it is common to refer to the intensity of the retardation pictured in the GDx-VCC printout as correlating with nerve fiber layer thickness, it is implicit that this is only in normal retinal tissue; one of the strengths of GDx is that it would seem to denote the quantitative distribution of the nerve fiber regardless of thickness due to edema [67].

Notwithstanding these differences, we have confirmed that OCT and GDx metrics yield very similar findings 
with respect to visual loss in patients with MS who have ON [17]. Like OCT, GDx produces measures that correlate with performance on low-contrast letter acuity and with changes in visual field; however, GDx can additionally detect abnormalities even when they are highly restricted to a particular distribution.

OCT, however, is also undergoing improvements. The new high-resolution OCT devices, such as the Cirrus OCT, offer automated disc centering and have longitudinal co-registration to minimize scan-to-scan variability. This device also corrects for eye movement, which will reduce retinal slip and image quality degradation. The high image acquisition speeds afforded by the Fourier domain instruments enable the generation of highdefinition OCT images with increased numbers of transverse pixels and improved coverage of the retina, as well as the acquisition of 3-dimensional OCT (3D-OCT) data sets. 3D-OCT imaging is especially promising because projection image data can be summed to provide a virtual fundus image, which enables precise and reproducible registration of individual OCT images to fundus features $[68,69]$. Taken together, these features promise to improve the reproducibility of RNFL thickness measurements and other morphometric measurements. Furthermore, 3D-OCT data can be processed to generate virtual circumpapillary scans, which can be registered to retinal features during post-processing (FIG. 5). Volumetric 3DOCT data also provide comprehensive information on the optic disc. RNFL-thickness maps (analogous to retardance maps from the GDx) or topographic maps (similar to those from HRT) can be generated [69].

Some important issues remain to be addressed. Different OCT instruments have different measurement protocols as well as different data analysis methods, so careful quantitative studies must be performed to compare morphometry results and to establish consistent normative baselines. In addition, questions remain about which protocols or visualization methods are best suited for a given application. The improved visualization and performance of new OCT technology suggests that it will have an increasingly important role in assessing the processes of axonal and neuronal degeneration in neurological disease in general and for MS in particular.

\section{ROLE FOR OCT IN DISTINGUISHING OTHER OPTIC NEUROPATHIES}

For the neurologist, separation of acute demyelinating ON from other causes of optic nerve dysfunction or visual loss is essential. The emergence of OCT technologies has enabled clinicians to perform measurements of RNFL and macular thickness that complement the standard medical history and clinical examination in distinguishing forms of acute optic neuropathy and retinal disease [24-28]. The age of onset of $\mathrm{ON}$ is typically between 20 and 50 years, and ON occurs threefold more frequently in women (Table 2) [55]. Visual loss in ON is most often unilateral in adults, although a bilateral presentation may occur more frequently in children. The temporal profile of the visual loss and subsequent visual recovery are important features of ON. Patients with typical ON usually lose vision over 7-10 days. In fact, progression of visual loss beyond 2 weeks is unusual [70]. Pain is an important feature of ON, and its absence should raise a question of whether the patient has an alternative etiology for their optic neuropathy. In the Optic Neuritis Treatment Trial (ONTT), $>90 \%$ of patients had pain, particularly with eye movements [71]. The pain usually persists for $1-$ 2 weeks. Finally, some recovery of vision should occur within 30 days of onset of ON. Clinical examination of patients with ON reveal reduced acuity in one eye, and an afferent pupillary defect is almost always present (unless a matched conduction defect is present in the fellow eye). There is impaired color vision, particularly to red-green objects, but occasionally to blue ones. Visual field defects are common, and almost any type of field defect may occur in ON. Diffuse visual field loss and central scotomas are common in typical $\mathrm{ON}$, particularly early in the course. Altitudinal defects are less commonly observed in $\mathrm{ON}$ and should raise the possibility of anterior ischemic optic neuropathy (AION) when optic disc swelling is present. In acute ON, optic disc swelling occurs in one-third of patients. The remainder of patients with $\mathrm{ON}$ will have retrobulbar inflammation in which the optic nerve head will initially appear normal. Features of atypical ON include the presence of neuroretinitis (swollen optic nerve head with retinal inflammation or exudates), a markedly swollen nerve, retinal hemorrhages, retinal exudates, and the presence of no-light-perception vision at onset [55]. Patients with these features have a lower risk of developing MS, particularly when a baseline brain MRI is normal [55, 72-74]. The distinction of ON from other causes of optic neuropathy is primarily based on the clinical features outlined above. The following are entities that commonly appear in the differential diagnosis of ON, with emphasis on OCT findings.

\section{Non-arteritic anterior ischemic optic neuropathy}

The main differential for $\mathrm{ON}$ is non-arteritic anterior ischemic optic neuropathy (NAION). There are no specific diagnostic tests for NAION and the diagnosis remains a clinical one. Typically, patients with NAION are older than 50 years, have no pain and, in almost all cases, the affected optic nerve is swollen acutely. Table 2 lists features that distinguish ON from NAION. One important characteristic of NAION is a small or absent cup in the fellow eye. In most OCT studies, NAION 
Table 2. Clinical Features that Distinguish Acute Demyelinating Optic Neuritis from Non-Arteritic Anterior Ischemic Optic Neuropathy

\begin{tabular}{|c|c|c|}
\hline Clinical features & Acute demyelinating optic neuritis $(\mathrm{ON})^{*}$ & $\begin{array}{l}\text { Non-arteritic anterior ischemic optic } \\
\text { neuropathy (NAION) }\end{array}$ \\
\hline Age, sex & 20-50 years; typically women $(3: 1)$ & $>50$ years; men or women \\
\hline Pain & $\begin{array}{l}\text { Present in }>90 \% \text {; exacerbated by eye movement; } \\
\text { resolves within 1 week }\end{array}$ & $\begin{array}{l}\text { Uncommon }(<10 \%) \text {; headache occurs in } \\
\text { temporal arteritis }\end{array}$ \\
\hline $\begin{array}{l}\text { Pattern of visual } \\
\text { loss }\end{array}$ & $\begin{array}{l}\text { Progression over hours to days, } 7-10 \text { days maximum; } \\
\text { unilateral in adult patients; signs of optic neuropathy } \\
\text { (visual acuity, color vision, and visual field defects) }\end{array}$ & $\begin{array}{l}\text { Sudden onset, often noted upon awakening } \\
\text { unilateral in adult patients; signs of optic } \\
\text { neuropathy (visual acuity, color vision, } \\
\text { and visual field defects) }\end{array}$ \\
\hline $\begin{array}{l}\text { Optic disc } \\
\text { appearance }\end{array}$ & $\begin{array}{l}\text { Optic disc swelling in one-third of patients, retrobulbar } \\
\text { in remainder; absence of hemorrhages/exudates }\end{array}$ & $\begin{array}{l}\text { Optic disc swelling, sectoral involvement, } \\
\text { hemorrhages; fellow eye disc crowded } \\
\text { with small or no cup }\end{array}$ \\
\hline Visual field defects & $\begin{array}{l}\text { Typically central, but may be arcuate (radiate from } \\
\text { physiologic blind spot); cecocentral (involve central } \\
\text { vision and physiologic blind spot); hemianopic } \\
\text { (involve area to right or left of vertical) }\end{array}$ & $\begin{array}{l}\text { Typically altitudinal (involve area above } \\
\text { or below horizontal) }\end{array}$ \\
\hline $\begin{array}{l}\text { Pattern of visual } \\
\text { recovery }\end{array}$ & $\begin{array}{l}\text { Begins within } 2-4 \text { weeks, generally favorable overall } \\
\text { ( } 20 / 20 \text { or better in } 75 \% \text { with baseline normal vision) }\end{array}$ & $\begin{array}{l}\text { Modest improvement over months in } \\
\text { approximately } 40 \% \text { (by } 3 \text { lines or more } \\
\text { on Snellen chart) }\end{array}$ \\
\hline \multirow[t]{3}{*}{ OCT findings } & Acute retrobulbar: Normal RNFL & Acute phase: Elevated RNFL, decreased \\
\hline & Acute papillitis: Elevated RNFL & cup to disc ratio in fellow eye \\
\hline & $\begin{array}{l}\text { Chronic ( }>3 \text { months): reduced RNFL thickness } \\
\text { by } 20-40 \% \text { in majority of patients }\end{array}$ & $\begin{array}{l}\text { Chronic phase: Reduced RNFL in the } \\
\text { majority of patients }\end{array}$ \\
\hline
\end{tabular}

*Absence of these typical features should suggest other causes of inflammatory optic neuropathy or alternative diagnoses, such as sarcoidosis, systemic lupus and other vasculitides, paraneoplastic disease, syphilis, Lyme disease, and Bartonella henselae infection (associated with cat scratch neuroretinitis), ischemic optic neuropathy, or Leber's hereditary optic neuropathy.

patients have smaller optic cups than controls [75-79]. The reduction in cup size appears to represent an anatomical risk factor for the development of NAION. There have been conflicting results regarding the reduction of total disc area in patients with NAION, with some studies showing a decrease in disc area size [79-81]. Variability in these observations may reflect differences in the technology used to measure disc area, with some studies using OCT technology while others used the HRT.

Following the acute injury, patients with NAION may show significant decreases in RNFL thickness in all quadrants, and these findings correlate well with visual field abnormalities [76, 77]. However, other studies have demonstrated a tendency of NAION to involve the superior quadrant of RNFL, particularly in those patients with inferior altitudinal loss $[78,79]$. In the acute phase, the thickness of the RNFL can be dramatically increased to nearly double the values seen in the unaffected fellow eye $[78,79]$. In one study of patients with NAION, arteritic anterior ischemic optic neuropathy (AAION) and open angle glaucoma, patients with open angle glaucoma had greater cup size and thinning of the neuroretinal rim than those with NAION and AAION. In comparing patients with AAION and NAION and controlling for the degree of visual field loss, patients with AAION had greater cup volume [80, 81].

\section{Neuromyelitis optica}

Patients with severe visual acuity loss (worse than 20/200) have an increased probability of having testing positive for NMO antibody. In fact, the visual prognosis for patients with severe acuity loss is far worse for those who are NMO positive $(>60 \%$ are $20 /$ 200 or less in 1 eye within 5 years) [82]. Patients with NMO usually have a greater degree of RNFL loss than those with $\mathrm{ON}$ and control patients. In one study, the average RNFL thickness for eyes of NMO patients was $63 \mu \mathrm{m}$ compared to $88 \mu \mathrm{m}$ for $\mathrm{ON}$ eyes and 102 $\mu \mathrm{m}$ for controls [83]. Using linear regression analysis, the authors of this study determined that a diagnosis of NMO was associated with a $24-\mu \mathrm{m}$ greater RNFL loss than that observed for those patients with $\mathrm{ON}$ associated with relapsing remitting MS. These findings also correlated well with more severe visual loss in the NMO group. Finally, NMO eyes may have a more diffuse loss of RNFL compared to the temporal (papillomacular bundle) nerve fiber loss that predominates with $\mathrm{ON}$ in the setting of relapsing remitting MS.

\section{Leber's hereditary optic neuropathy}

Leber's optic neuropathy (LHON) is a hereditary optic nerve condition that predominately occurs in young men [84]. The visual loss in LHON is painless, providing an 
important distinction from demyelinating ON. Visual loss is often severe, and the fellow eye may become involved in a matter of weeks after the initial presentation. The common point mutations associated with LHON include 11778, 14484, and 3460 [84]. The optic nerve may appear elevated (pseudo-swelling), and teleangiectatic vessels may be evident in approximately $50 \%$ of patients.

The OCT findings of LHON demonstrate an interesting sequence of RNFL involvement [85]. In a recent study, a significant increase of RNFL thickness was noted in the temporal and inferior quadrants in the presymptomatic state to disease onset. At 3 months, the thickening was more evident in the superior and nasal quadrants, presumably as the temporal and inferior quadrants (responsible for papillomacular function) began to atrophy. At 9 months, all but the nasal quadrants showed significant thinning. The reason why the inferior quadrant may be affected early on is unclear, but structural changes at the lamina cribosa similar to what occurs in glaucoma may be responsible. It is notable that the retinal nerve fiber swelling of the inferior quadrant followed by that of the superior quadrant may take months to evolve. The occurrence of marked acute vision loss with relatively slow changes in the RNFL in a distinct pattern may provide a clue about the pathogenesis of LHON. Other studies have examined unaffected carriers to detect the earliest changes of LHON and to assess those risk factors that might be associated with development of this disorder. In one OCT study, the mean optic disc area was significantly larger in the carriers than in the affected patients, suggesting a protective role for those patients that have large disc areas [86]. In another study, thickening of the temporal fibers was detected in unaffected carriers, suggesting preferential involvement of the papillomacular bundle in subclinical LHON [87]. The visual prognosis of LHON may vary depending on the specific mitochondrial mutation. Seo et al. [88] found that those patients harboring the 11778 mutation had significantly more RNFL loss than those with the 14484 mutation. This observation appears to correspond with low visual recovery rate associated with the 11778 mutation.

\section{Other inflammatory disorders of the optic nerve}

A number of other inflammatory optic neuropathies should be considered when the visual loss is bilateral or associated with hemorrhages, retinal exudates, or uveitis. These include sarcoid, syphilis, Lyme disease, lupus, cat scratch fever, and viral etiologies. In the routine case of demyelinating $\mathrm{ON}$, serological tests are of limited diagnostic value. In patients with atypical or systemic features, serological studies for sarcoid, syphilis, Lyme disease, Sjogren's disease, toxoplamosis, cat scratch fever, West Nile virus, human immunodeficiency virus infection, and herpes infection should be considered [89].

\section{Toxic optic neuropathies}

Toxic or nutritional optic neuropathies are suggested when there is bilateral, painless, and symmetrical visual loss. The optic nerve head may appear to be normal or atrophic. Classically, toxic or nutritional optic neuropathies are associated with centrocecal scotomas (central scotomas that connect to the normal blind spot). B12 deficiency and ethambutol toxicity are two common causes. The OCT of toxic optic neuropathy usually demonstrates a bilateral and progressive loss of RNFL. We recently noted that patients with copper deficiency may show retinal nerve fiber loss [90]. Since the human diet requires only a trace amount of copper, it may take decades before the changes are noted. We have seen patients with copper deficiency after gastric surgery for ulcerative disease as well as bariatric surgery for morbid obesity.

\section{Compressive and infiltrative optic neuropathies}

When visual loss persists beyond several weeks, the possibility of a tumor or infiltrative process should be considered. The presence of optic atrophy at the initial presentation of suspected $\mathrm{ON}$ should also raise the possibility of a tumor or a prior sub-clinical episode of ON. Progressive visual loss, optic atrophy, and optic nerve head shunt vessels should suggest the possibility of an optic nerve sheath meningioma or a pituitary region tumor. Bilateral visual loss in a patient with known cancer or suspected cancer raises the possibility of a paraneoplastic process.

The visual outcome following surgical treatment of a pituitary adenoma can be hard to predict. OCT in several small studies has been useful in predicting visual outcome following the treatment of pituitary adenomas [91, 92]. In a study of 19 patients, a greater RNFL thickness prior to treatment increased the probability of visual recovery following treatment (17 surgical and 2 pharmacological treatments). For each increase in RNFL by $1 \mu \mathrm{m}$, the odds of complete recovery were multiplied by 1.29 [91]. A strong correlation was found between the initial visual field defect and the mean RNFL thickness. The prognostic value of RNFL thickness was independent from the effects of age and duration of symptoms [91]. These findings suggest that RNFL thinning is the result of RGC cell axonal degeneration and the product of compression of the anterior visual pathway fibers by the underlying chiasmal mass lesion. The permanent damage of the RNFL correlates with the persistence of the visual field defect. However, since the vast majority of patients in this study showed an improved visual field following surgery, the presence of RNFL thinning should not preclude surgical therapy. 


\section{Role for OCT in distinguishing retinal disorders}

A variety of retinal conditions may initially be confused with demyelinating ON [24-28]. Fortunately, there are often distinctive retinal findings that separate these entities, which may include the following:

1. Central serous chorioretinopathy tends to occur in young men who have a type-A personality. The visual loss is acute and painless. There is a retinal detachment in the region of the macula that produces central visual field loss. Classically, there is a break in the retinal pigment epithelium that results in fluid passing into the subretinal space and a retinal detachment. The process can be well documented by OCT [93]. Fluid under the macula may resolve spontaneously and thus mimic the recovery of ON. Other retinal entities usually pose no major problems in the differential diagnosis of $\mathrm{ON}$ because they produce such distinctive retinal changes.

2. Retinal artery occlusion is distinguished by the presence of retinal whitening characteristic of infarction.

3. Retinal vein occlusion is distinguished by the presence of retinal hemorrhages, which are often peripheral, and dilated retinal veins.

The distinction between glaucomatous optic neuropathy and demyelinating $\mathrm{ON}$ is usually not difficult. Patients with glaucoma usually have progressive loss of vision, elevated intraocular pressures, and optic disc cupping. OCT is frequently used to monitor disease progression. The amount of RNFL loss can be variable in patients with glaucoma, with the greatest rates occurring in those with the higher baseline RNFL thickness. As one might expect, the greatest loss of RNFL occurs in the inferior temporal quadrant corresponding to the superior nasal field [94]. However, in patients with angle closure glaucoma, the separation may be more complicated. Patients with angle closure glaucoma present with acute visual loss and pain. These patients have a small anterior chamber, and the affected eye is often red during the acute attack. Occasionally, patients with open angle glaucoma will present with episodic or acute visual loss. Optic nerve head swelling is absent, but there may be a splinter hemorrhage at the optic disc margin.

\section{OCT IN NEUROLOGIC DISEASE}

\section{Alzheimer's disease}

Early visual aberrations in contrast sensitivity, depth, and motion perception are symptomatic of Alzheimer's disease (AD). Clinical studies of the optic nerve head and RNFL using photographic methods have suggested that optic neuropathies with RNFL abnormalities are characteristic of AD. Iseri et al. [95] addressed the conflicting evidence on whether AD and the loss of RGC are linked. Their results showed that the peripapillary RNFL thick- ness in all quadrants in patients with $\mathrm{AD}$ was thinner than that in controls $(87.7 \pm 23.8 v s 113.2 \pm 6.8 \mu \mathrm{m})$. Total macular volumes and Mini-Mental State Exam scores correlated significantly $(r=0.70, p=0.006)$, while no significant difference was found for VEPs. These results suggest that peripapillary RNFL thickness and macular volume are decreased in patients with $\mathrm{AD}$ disease and that the macular volume relates to the severity of cognitive impairment because decreases in macular volume were positively correlated with decreases in Mini-Mental State Exam scores.

By comparison to the more advanced $\mathrm{AD}$ patients studied by Iseri et al. [95], Berisha et al. [96] focused on patients classified as having mild to moderate dementia. This shift in patient population was made in order to determine where the earliest thinning occurs in patients with AD. Their results indicate that retinal damage due to $\mathrm{AD}$ may begin in the superior quadrant $(92.2 \pm 21.6 \mu \mathrm{m} v s \quad 113.6 \pm 10.7 \mu \mathrm{m}$ for controls, $p=0.02$ ), instead of just overall thinning within all four quadrants. These authors found no statistically significant RNFL thinning in the temporal, nasal, or inferior quadrants. These findings also suggest that neuronal degeneration in the retina may be related to field deficits in $\mathrm{AD}$ and that narrowing of retinal venous blood column diameter and a reduction in retinal blood flow rate occur in these patients. However, there was no correlation between RNFL thickness and retinal blood flow.

\section{Parkinson's disease}

Visual impairment is a commonly exhibited nonmotor feature of Parkinson's disease (PD). In particular, the loss of foveal vision has been linked to retinopathy in patients with PD. The use of OCT has allowed for a better understanding of morpohological changes in the retinal neurons [97]. Recent studies have shown that while visual acuity testing suggests only minimal affects in best-corrected PD in comparison to controls, PD patients do lose foveal contrast sensitivity to patterns. It is now possible to determine the functional deficit of the neural retina and retinal output of PD patients through the use of electroretinogram and pattern ERG. Within the dopamine-deficient retina, electroretinogram and pattern electroretinogram results suggest a lack of ganglion cell receptive field organization. Furthermore, OCT can be used to quantify morphology changes of retina in patients with PD. RNFL thinning in PD patients has also been reported, although the results are mixed; in addition, this thinning may be more reflective of RGC and other neuronal loss. Bodis-Wollner's [97] results indicate that the inner retina is thinned in relatively early PD and that the loss of RNFL in PD is not secondary to increases in intraocular pressure. In addition to the thinning of the ganglion cell layer, results suggest a thinning of the macular region of the inner retina.

Evaluations of the inner retinal layer and outer retinal layer were also made by Hajee et al. [98]. Their results 
indicate that patients had increased thinning in early PD and that RNFL loss was not the result of intraocular pressures. The outer retinal layer and inner retinal layer thicknesses were both analyzed, and the thickness of the superior inner retinal layer was lower in patients with $\mathrm{PD}$ $(88.8 \pm 11.3 \mu \mathrm{m})$ than in control eyes $(103.5 \pm 24.3 \mu \mathrm{m}$, $p=0.01)$. When compared with healthy eyes, the retinas of PD patients were approximately $15 \%$ thinner. While Inzelberg et al. [99] reported a stronger effect in the inferior peripapillary quadrant, more recent findings suggest that thickness in the superior and inferior macular regions are approximately equal [98].

\section{Friedreich ataxia}

Integrative approaches to understanding the visual function of patients with Friedreich ataxia (FRDA) are necessary for better patient care. In particular, although common in patients with mitochondrial disease, optic neuropathy in FRDA patients is not well understood. Using a variety of techniques to assess the visual pathway, Fortuna et al. [100] evaluated 26 patients with FRDA. All patients showed a reduced RNFL thickness with varying degrees of severity of axonal loss. Visual fields, OCT, and electrophysiology were used in tandem to ascertain the various states of the visual pathway. Fortuna et al.'s study showed a diffuse and progressive pattern of fiber loss, beginning with the periphery and becoming concentrically worse. All patients showed significantly reduced RNFL thickness in all quadrants and on average $(76.0 \pm 12.0$ vs $100.0 \pm 8.9 \mu \mathrm{m} ; p<$ 0.0001 ). Given the wide variability in clinical presentation, the study also addressed two cases in which patients had sudden bilateral loss of vision similar to clinical course of LHON. Uniquely in these cases, these heterozygote patients were the most severely affected with visual loss. Their OCT results showed average RNFL thickness values of $76.0 \pm 12.0 \mu \mathrm{m}$ compared with $100 \pm$ $8.9 \mu \mathrm{m}$ in healthy controls $(p<0.0001)$. This study also shows that FRDA has a unique pattern of visual loss and pathological mechanism unlike that of non-syndromic mitochondrial optic neuropathies. However, as illustrated in the case of the two patients who experienced visual loss spontaneously, LHON mimicking can occur within patients with Friedreich's Ataxia. Thus, within FRDA there is a variation of clinical expression with respect to RGC degeneration to sudden papillomacular RGC loss.

\section{CONCLUSION}

Rapid advancements in OCT technologies have allowed for improved visualization of the retina. Highresolution Fourier domain OCT has allowed for rapid imaging of the retina as well as 3-dimensional imaging and improved volumetric measurements [69]. OCT has now been incorporated into a number of clinical trials for MS, emphasizing the role of the anterior visual pathway as a model for testing new therapies, particularly those involving neuroprotection. The results of studies have suggested that losses of axons and ganglion cells may be correlated with MRI measures that reflect more global aspects of neuronal and axonal degeneration. Additionally, the capacity for OCT to measure both RNFL and macular thickness makes these measures attractive structural markers to help distinguish MS subtypes and understand disease progression. The incorporation of OCT into the study of neurodegenerative disorders will provide new insights into a more global role for this technology in neurologic disease.

Acknowledgments: This work was supported by the National Multiple Sclerosis Society (RG 4212-A-4; TR 3760A-3); NIH/ National Eye Institute (R01 EY 019473; K24 EY 018136); the DAD's Foundation, and the McNeill Foundation. We are grateful to Dr. Steven Galetta for helpful input and expertise regarding this manuscript.

\section{REFERENCES}

1. Trapp BD, Peterson J, Ransohoff RM, et al. Axonal transection in the lesions of multiple sclerosis. N Engl J Med 1998;338:278-285.

2. Evangelou N, Konz D, Esiri MM, et al. Size-selective neuronal changes in the anterior optic pathways suggest a differential susceptibility to injury in multiple sclerosis. Brain 2001;124:18131820.

3. DeLuca GC, Williams K, Evangelou N, et al. The contribution of demyelination to axonal loss in multiple sclerosis. Brain 2006;129:1507-1516.

4. Sepulcre J, Goñi J, Masdeu JC, et al. Contribution of white matter lesions to gray matter atrophy in multiple sclerosis: evidence from voxel-based analysis of T1 lesions in the visual pathway. Arch Neurol. 2009;66:173-179.

5. Frohman EM, Fujimoto JG, Frohman TC, et al. Optical coherence tomography: a window into the mechanisms of multiple sclerosis. Nat Clin Pract Neurol 2008;4:664-675.

6. Parisi V, Manni G, Spadaro M, et al. Correlation between morphological and functional retinal impairment in multiple sclerosis patients. Invest Ophthalmol Vis Sci 1999;40:2520-2527.

7. Trip SA, Schlottmann PG, Jones SJ, et al. Retinal nerve fiber layer axonal loss and visual dysfunction in optic neuritis. Ann Neurol 2005;58:383-391.

8. Costello F, Coupland S, Hodge W, et al. Quantifying axonal loss after optic neuritis with optical coherence tomography. Ann Neurol 2006;59:963-969.

9. Fisher JB, Jacobs DA, Markowitz CE, et al. Relation of visual function to retinal nerve fiber layer thickness in multiple sclerosis. Ophthalmology 2006;113:324-332.

10. Cheng H, Laron M, Schiffman JS, Tang RA, Frishman LJ. The relationship between visual field and retinal nerve fiber layer measurements in patients with multiple sclerosis. Invest Ophthalmol Vis Sci 2007;48;5798-5805.

11. Pulicken M, Gordon-Lipkin E, Balcer LJ, et al. Optical coherence tomography and disease subtype in multiple sclerosis. Neurology 2007;69:2085-2092.

12. Costello F, Hodge W, Pan YI, et al. Differences in retinal nerve fiber layer atrophy between multiple sclerosis subtypes. J Neurol Sci 2009;281:74-79.

13. Henderson AP, Trip SA, Schlottmann PG, et al. An investigation of the retinal nerve fibre layer in progressive multiple sclerosis using optical coherence tomography. Brain 2008;131:277-287. 
14. Gordon-Lipkin E, Chodkowski B, Reich DS, et al. Retinal nerve fiber layer is associated with brain atrophy in multiple sclerosis. Neurology 2007;69:1603-1609.

15. Sepulcre J, Murie-Fernandez M, Salinas-Alaman A, et al. Diagnostic accuracy of retinal abnormalities in predicting disease activity in MS. Neurology 2007;68:1488-1494.

16. Cettomai D, Pulicken M, Gordon-Lipkin E, et al. Reproducibility of optical coherence tomography in multiple sclerosis. Arch Neurol 2008;65:1218-1222.

17. Zaveri M, Conger A, Salter A, et al. Retinal imaging by laser polarimetry corroborates optical coherence tomography evidence of axonal degeneration in multiple sclerosis. Arch Neurol 2008;65:924-928.

18. Salter AR, Conger A, Frohman TC, et al. Retinal architecture predicts pupillary reflex metrics in MS. Mult Scler 2008;15:479486.

19. Pueyo V, Ara JR, Almarcegui C, et al. Sub-clinical atrophy of the retinal nerve fibre layer in multiple sclerosis. Acta Ophthalmol 2010;88:748-752.

20. Costello F, Hodge W, Pan YI, Metz L, Kardon RH. Retinal nerve fiber layer and future risk of multiple sclerosis. Can J Neurol Sci 2008;35:482-487.

21. Burkholder BM, Osborne B, Loguidice MJ, et al. Macular volume by optical coherence tomography as a measure of neuronal loss in multiple sclerosis. Arch Neurol 2009;66:1366-1372.

22. Kolappan M, Henderson APD, Jenkins TM, et al. Assessing structure and function of the afferent visual pathway in multiple sclerosis and associated optic neuritis. J Neurol 2009;256:305-319.

23. Barkhof F, Calabresi P, Miller DH, Reingold SC. Imaging outcomes for neuroprotection and repair in multiple sclerosis trials. Nat Rev Neurol 2009;5:256-266.

24. Jindahra P, Hedges TR, Mendoza-Santiesteban CE, Plant GT. Optical coherence tomography of the retina: applications in neurology. Curr Opin Neurol 2010;23:16-23.

25. Lameril C, Newman N, Biousse V. The use of optical coherence tomography in neurology. Rev Neurol Dis 2009;6:E105-120.

26. Sakata LM, DeLeon-Ortega J, Sakata V, Girkin CA. Optical coherence tomography of the retina and optic nerve - a review. Clin Exp Ophthalmol 2009;37:90-99.

27. Glisson CC, Galetta SL. Nonconventional optic nerve imaging in multiple sclerosis. Neuroimag Clin N Am 2009;19:71-79.

28. Kallenbach K, Frederiksen J. Optical coherence tomography in optic neuritis and multiple sclerosis: a review. Eur J Neurol 2007:14:841-849.

29. Frohman E, Costello F, Zivadinov R, et al. Optical coherence tomography in multiple sclerosis. Lancet Neurol 2006;5:853-863.

30. Burkholder BM, Osborne B, Loguidice MJ, et al. Macular volume determined by optical coherence tomography as a measure of neuronal loss in multiple sclerosis. Arch Neurol 2008;66:13661372.

31. Talman LS, Bisker ER, Sackel DJ, et al. Longitudinal study of vision and retinal nerve fiber layer thickness in multiple sclerosis. Ann Neurol 2010;67:749-760.

32. Syc SB, Warner CV, Hiremath GS, et al. Reproducibility of highresolution optical coherence tomography in multiple sclerosis. Mult Scler 2010;16:829-839.

33. Traboulsee A, Dehmeshki J, Peters KR, et al. Disability in multiple sclerosis is related to normal appearing brain tissue MTR histogram abnormalities. Mult Scler 2003;9:566-573.

34. Frohman EM, Zhang H, Kramer PD, et al. MRI characteristics of the MLF in MS patients with chronic internuclear ophthalmoparesis. Neurology 2001;57:762-768.

35. Frohman EM, Frohman TC, O'Suilleabhain P et al. Quantitative oculographic characterization of internuclear ophthalmoparesis in multiple sclerosis: the versional dysconjugacy index $\mathrm{Z}$ score. J Neurol Neurosurg Psychiatry 2002;73:51-55.

36. Fox RJ, McColl RW, Lee JC, et al. A preliminary validation study of diffusion tensor imaging as a measure of functional brain injury. Arch Neurol 2008;65:1179-1184.

37. Huang D, Swanson EZ, Lin CP, et al. Optical coherence tomography. Science 1991;254:1178-1181.

38. Swanson EA, Izatt JA, Hee MR, et al. In vivo retinal imaging by optical coherence tomography. Opt Lett 1993;18:1864-1866.
39. Fercher AF, Hitzenberger CK, Drexler W, et al. In vivo optical coherence tomography. Am J Ophthalmol 1993;116:113-114.

40. Hee MR, Puliafito CA, Wong C, et al. Optical coherence tomography of the human retina. Arch Ophthalmol 1995;113:325-332.

41. Puliafito CA, Hee MR, Lin CP, et al. Imaging of macular diseases with optical coherence tomography. Ophthalmology 1995;102:217229.

42. Drexler W and Fujimoto JG. State-of-the-art retinal optical coherence tomography. Prog Retin Eye Res 2008;27:45-88.

43. de Boer JF, Cense B, Park BH, et al. Improved signal-to-noise ratio in spectral-domain compared with time-domain optical coherence tomography. Opt Lett 2003;28:2067-2069.

44. Leitgeb R, Hitzenberger A, Fercher C. Performance of Fourier domain $v$ time domain optical coherence tomography. Opt Express 2003;11:889-894

45. Wojtkowski M, Leitgeb R, Kowalczyk A, Bajraszewski T, Fercher $\mathrm{AF}$. In vivo human retinal imaging by Fourier domain optical coherence tomography. J Biomed Opt 2002;7:457-463.

46. Nassif N, Cense B, Park BH, et al. In vivo human retinal imaging by ultrahigh-speed spectral domain optical coherence tomography. Opt Lett 2004;29:480-482.

47. Wojtkowski M, Srinivasan V, Ko T, Fujimoto J, Kowalczyk A, Duker J. Ultrahigh-resolution, high-speed, Fourier domain optical coherence tomography and methods for dispersion compensation. Opt Express 2004;12:2404-2422.

48. Choma MA, Sarunic M, Yang C, Izatt J. Sensitivity advantage of swept source and Fourier domain optical coherence tomography. Opt Express 2003;11:2183-2189.

49. Keeler CR. The ophthalmoscope in the lifetime of Hermann von Helmholtz. Arch Ophthalmol 2002;120:194-201.

50. Frisén L and Hoyt WF. Insidious atrophy of retinal nerve fibers in multiple sclerosis. Funduscopic identification in patients with and without visual complaints. Arch Ophthalmol 1974;92:91-97.

51. Kerrison JB, Flynn T, Green WR. Retinal pathologic changes in multiple sclerosis. Retina 1994;14:445-451.

52. Frohman EM, Costello F, Stüve O, et al. Modeling axonal degeneration within the anterior visual system: implications for demonstrating neuroprotection in multiple sclerosis. Arch Neurol 2008;65:26-35.

53. Ikuta F, Zimmerman HM. Distribution of plaques in seventy autopsy cases of multiple sclerosis in the United States. Neurology 1976;26:26-28.

54. Toussaint D, Périer O, Verstappen A, Bervoets S.. Clinicopathological study of the visual pathways, eyes, and cerebral hemispheres in 32 cases of disseminated sclerosis. J Clin NeuroOphthalmol 1983;3:211-220.

55. Balcer LJ. Clinical practice. Optic neuritis. N Engl J Med 2006;354:1273-1280

56. Frohman EM, Frohman TC, Zee DS, McColl R, Galetta S. The neuro-ophthalmology of multiple sclerosis. Lancet Neurol 2005;4:111-121.

57. Kupersmith MJ, Alban T, Zeiffer B, Lefton D. Contrast-enhanced MRI in acute optic neuritis: relationship to visual performance. Brain 2002;125:812-822.

58. Hickman SJ, Toosy AT, Jones SJ, et al. A serial MRI study following optic nerve mean area in acute optic neuritis. Brain 2004;127:2498-2505

59. Wu GF Schwartz ED, Lei T, et al. Relation of vision to global and regional brain MRI in multiple sclerosis. Neurology 2007;69:21282135.

60. Frohman EM, Racke MK, Raine CS, et al. Multiple sclerosis - the plaque and its pathogenesis. N Engl J Med 2006;354:942-955.

61. Kanamori A, Escano MF, Eno A, et al. Evaluation of the effect of aging on retinal nerve fiber layer thickness measured by optical coherence tomography. Opthalmologica 2003;217:273-278.

62. Balcer LJ, Baier ML, Cohen JA, et al. Contrast letter acuity as a visual component for the Multiple Sclerosis Functional Composite. Neurology 2003;61:1367-1373.

63. Baier ML, Cutter GR, Rudick RA, et al. Low-contrast letter acuity testing captures visual dysfunction in patients with multiple sclerosis. Neurology 2005;64:992-995.

64. Grazioli E, Zivadinov R, Weinstock-Guttman B, et al. Retinal nerve fiber layer thickness is associated with brain MRI outcomes in multiple sclerosis. J Neurol Sci 2008;268:12-17. 
65. Green A, McQuaid S, Hauser SL, Allen IV, Lyness R. Ocular pathology in multiple sclerosis: retinal atrophy and inflammation irrespective of disease duration. Brain 2010;133:1591-1601.

66. Naismith RT, Tutlam NT, Xu J, Shepherd JB, Klawiter EC, Song SK, Cross AH. Optical coherence tomography is less sensitive than visual evoked potentials in optic neuritis. Neurology 2009;73:46-52.

67. Banks MC et al. Scanning laser polarimetry of edematous and atrophic optic nerve heads. Arch Ophthalmol 2003;121:484-490.

68. Jiao $\mathrm{S}$ et al. Simultaneous acquisition of sectional and fundus ophthalmic images with spectral-domain optical coherence tomography. Opt Express 2005;13:444 452.

69. Wojtkowski $\mathrm{M}$ et al. Three-dimensional retinal imaging with highspeed ultrahigh-resolution optical coherence tomography. Ophthalmology 2005;112:1734-1746.

70. Beck RW, Cleary PA, Anderson MM, et al. A randomized, controlled trial of corticosteroids in the treatment of acute optic neuritis. N Engl J Med 1992;326:581-588.

71. Optic Neuritis Study Group: The clinical profile of optic neuritis. Experience of the Optic Neuritis Treatment Trial. Arch Ophthalmol 1991;109:1673-1678.

72. Optic Neuritis Study Group. High risk and low risk profiles for the development of multiple sclerosis within 10 years after optic neuritis: Experience of the Optic Neuritis Treatment Trial. Arch Ophthalmol 2003;121:944-949.

73. Optic Neuritis Study Group. The 5 year risk of multiple sclerosis after optic neuritis: Experience of the optic neuritis treatment trial. Neurology 1997;49:1404-1413.

74. Balcer LJ, Galetta SL. Optic neuritis. In: Conn's current therapy. Rakel RE, Bope ET (Eds.), Philadelphia: W.B. Saunders, pp 187190, 2004.

75. Ischemic Optic Neuropathy Decompression Trial Research Group. Optic nerve decompression surgery for nonarteritic anterior ischemic optic neuropathy (NAION) is not effective and may be harmful. JAMA 1995;273:625-632.

76. Chan CKM, Cheng ACO, Leung CKS et al. Quantitative assessment of optic nerve head morphology and retinal nerve fibre layer in non-arteritic anterior ischaemic optic neuropathy with optical coherence tomography and confocal scanning laser ophthalmoscopy. Br J Ophthalmol 2009;93:731-735.

77. Alasil T, Tan O, Hui Lu, AT et al. Correlation of Fourier domain optical coherence tomography retinal nerve fiber layer maps with visual fields in nonarteritic ischemic optic neuropathy. Ophthalmic Surg Lasers Imaging 2008;39:S71-S79.

78. Contreras I, Noval S, Rebolleda G et al. Follow-up of nonarteritic anterior ischemic optic neuropathy with optical coherence tomography. Ophthalmology 2007;114: 2338-2344.

79. Horowitz J, Fishelzon T, Rath EZ et al. Comparison of optic nerve head topography findings in eyes with non arteritic anterior ischemic optic neuropathy and eyes with glaucoma. Graefes Arch Clin Ophthalmol 2010;248:845-851.

80. Danesh-Meyer HV, Boland MV, Savino PJ et al. Optic disc morphology in open angle glaucoma compared with anterior ischemic optic neuropathies. Invest Ophthalmol Vis Sci 2010;51:2003-2010.

81. Contreras I, Rebolleda G, Noval S et al. Optic disc evaluation by optical coherence tomography in nonarteritic anterior ischemic optic neuropathy. Invest Ophthalmol Vis Sci 2007;48:4087-4092.

82. Wingerchuk DM, Hogancamp WF, O’Brien PC, Weinshenker BG. The clinical course of neuromyelitis optica (Devic's syndrome). Neurology 1999;53:1107-1114.
83. Ratchford JN, Quigg ME, Conger A et al. Optical coherence tomography helps differentiate neuromyelitis optica from MS optic neuropathies. Neurology 2009;73:302-308.

84. Newman NJ, Biousse V. Hereditary optic neuropathies. Eye 2004; $18: 114-160$.

85. Barboni P, Carbonelli M, Savini G et al. Natural history of Leber's hereditary optic neuropathy: Longitudinal analysis of the retinal nerve fiber layer by optical coherence tomography. Ophthalmology 2010;117:623-627.

86. Ramos CVF, Bellusci C, Savini G et al. Association of optic disc size with development and prognosis of Leber's hereditary optic neuropathy. Invest Ophthalmol Vis Sci 2009;50:16661674.

87. Savini G, Barboni P, Valentino ML et al. Retinal nerve fiber layer evaluation by optical coherence tomography in unaffected carriers with Leber's hereditary optic neuropathy mutations. Ophthalmology 2005; 112:127-131.

88. Seo JH, Hwang JM, Park SS. Comparison of retinal nerve fibre layer between 11778 and 14484 mutations in Leber's hereditary optic neuropathy. Eye 2010;24:107-111.

89. Schmalfuss IM, Dean CW, Sistrom C, Bhatti MT. Optic neuropathy secondary to cat scratch disease: Distinguishing MR imaging features from other types of optic neuropathies. AJNR Am J Neuroradiol 2010;26:1310-1316.

90. Pineles SL, Wilson CA, Balcer LJ et al. Combined optic neuropathy and myelopathy secondary to copper deficiency. Surv Ophthalmol 2010;55:386-392.

91. Danesh-Meyer HV, Papchenko T, Savino PJ et al. In vivo retinal nerve fiber layer thickness measured by optical coherence tomography predicts visual recovery after surgery for parachiasmal tumors. Invest Ophthalmol Visual Sci 2008;49:1879-1885.

92. Jacob M, Raverot G, Jounneau E et al. Predicting visual outcome after treatment of pituitary adenomas with optical coherence tomography. Am J Ophthalmol 2009;147:64-70.

93. Shinojima A, Hirose T, Mori et al. Morphologic findings in acute central serous chorioretinopathy using spectral domain optical coherence tomography with simultaneous angiography. Retina 2010;30:193-202.

94. Leung CK, Cheung CYL, Weinreb RN et al. Evaluation of retinal nerve fiber progression in glaucoma: A study on optical coherence tomography guided progression analysis. Invest Ophthalmol Vis Sci 2010;51:217-222.

95. Iseri PK, Atlinaş Ö, Tokay T, Yüksel N. Relationship between cognitive impairment and retinal morphological and visual functional abnormalities in Alzheimer disease. J Neuro-Ophthalmol 2006;26:18-24.

96. Berisha F, Feke GT, Trempe CL, McMeel JW, Schepens CL. Retinal abnormalities in early Alzheimer's disease. Invest Ophthalmol Vis Sci 2007;48:2285-2289.

97. Bodis-Wollner I. Retinopathy in Parkinson disease. J Neural Transm 2009;116:1493-1501.

98. Hajee ME, March WF, Lazzaro DR, et al. Inner retinal layer thinning in Parkinson disease. Arch Ophthalmol 2009;127:737741 .

99. Inzelberg R, Ramirez JA, Nisipeanu P, Ophir A. Retinal nerve fiber layer thinning in Parkinson disease. Vision Res 2004;44:27932797.

100. Fortuna F, Barboni P, Liguori R, et al. Visual system involvement in patients with Friedreich's ataxia. Brain 2009;132:116-123. 